\title{
Flares and non-potentiality of AR 11158
}

\author{
Qiao Song ${ }^{1}$, Jun Zhang ${ }^{1}$, Shuhong Yang ${ }^{1}$ and Yang Liu ${ }^{2}$ \\ ${ }^{1}$ Key Laboratory of Solar Activity, National Astronomical Observatories, \\ Chinese Academy of Sciences, Beijing 100012, China \\ email: qsong@nao.cas.cn \\ ${ }^{2}$ W. W. Hansen Experimental Physics Laboratory, \\ Stanford University, Stanford, CA 94305-4085, USA
}

\begin{abstract}
Five non-potential parameters are calculated to investigate the temporal and spatial variations of vector magnetic field in active region 11158. An area that had evident changes of the azimuth of the vector magnetic field was found. The sunspot rotation may lead to an increase of the non-potentiality. Rapid and prominent increases are found in the variations of unsigned helicity.
\end{abstract}

Keywords. Sun: flares, Sun: magnetic fields, Sun: photosphere

\section{Introduction}

Magnetic non-potentiality is a key to understanding flares and other solar activities. Five non-potential parameters, i.e., electric current, current helicity, source field, angular shear $\left(\theta_{a s}\right)$, and photospheric free energy (see Wang 1999 review), were calculated to study the temporal and spatial variations of active region (AR) 11158. The AR erupted the first X-class flare of Solar Cycle 24 and 3 M-class flares during five days. Vector magnetograms from the Helioseismic and Magnetic Imager (Schou et al. 2012) were used to quantify the photosphere vector magnetic field.

\section{Results}

Fig. 1a shows the vector magnetogram of AR 11158 before the X-class flare. The small area indicated by the box in the $\theta_{a s}$ map (Fig. 1b) has the opposite sign with its surrounding and it is co-spatial with a source region of a sunquake event (Kosovichev et al. 2011). As shown in Fig. 1c, an anticlockwise vortex around P2 becomes starkly evident during the flare in source field map. The vortex appeared about $15 \mathrm{~h}$ before the flare and it disappeared on the next day along with n8. As can be seen from the thick arrow and dash line in Fig. 1d, the average azimuth markedly changed due to the cancellation between $\mathrm{P} 2$ and $\mathrm{n} 8$. The total change of azimuth was $54^{\circ}$ with an average rate of $1.3^{\circ} \mathrm{h}^{-1}$.

The change of the unsigned helicity for the X-class flare and other three M-class flares are given in the upper line in Fig. 2. It had a fast and significant increase. The recovery of the increases took 3-4 h for the two bigger flares, while only took less than $2 \mathrm{~h}$ for the other two smaller flares. The change of unsigned magnetic flux, current, angular shear, and total free energy in region $\mathrm{C}$ are plotted in the lower line. The unsigned flux continued increasing and reached its peak about $3 \mathrm{~h}$ after the X-class flare, while the unsigned current had similar but temperate increases with helicity. Furthermore, the total free energy in region $\mathrm{C}$ had a rapid increase more than $10 \%$ during the $\mathrm{X}$-class flare while other flare regions had smaller increases. More details are described in Song et al. (2012). 


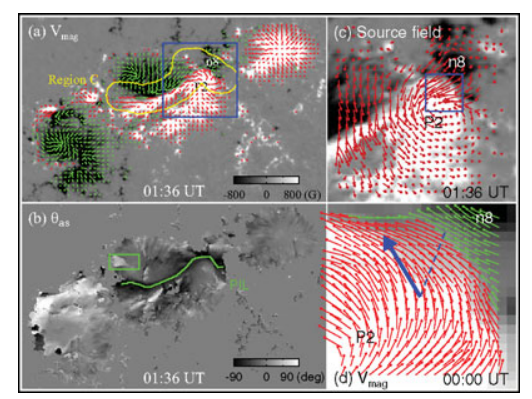

Figure 1. Vector magnetograms and non-potential parameter maps of AR 11158. The box in

(a) indicates the field of view (FOV) of (c) and the box in (c) shows the FOV of (d).
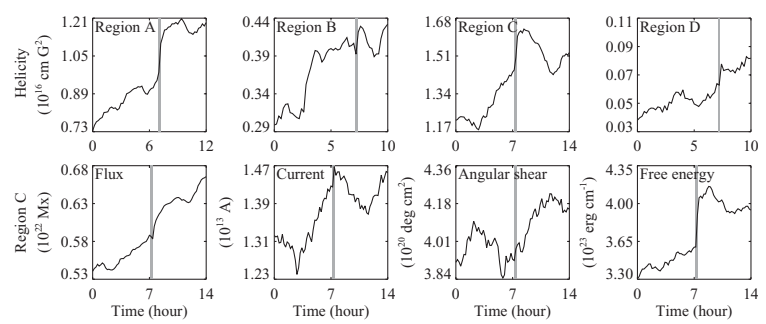

Figure 2. Change of non-potential parameters during flares. The gray shadow on each panel indicates the duration of the corresponding flare.

\section{Discussion and Summary}

The vortex in the source field is corresponding to the fast rotated sunspot with a time delay, which suggests that the sunspot rotation leads to an increase of the nonpotentiality. The sheared and twisted magnetic field may eventually cause the flare (Zhang et al. 2007). An area that had evident changes of the azimuth of the vector magnetic field was found near the magnetic polarity inversion line. Rapid and prominent increases were found in the variations of unsigned helicity during all four flares in their initial brightening regions. It may be due to that regions $\mathrm{A}$ and $\mathrm{C}$ covered the $\delta$-sunspot of the AR which contains a bigger, complex and pivotal magnetic structure for flares.

\section{Acknowledgements}

The work is supported by the National Basic Research Program of China under grant 2011CB811403, the National Natural Science Foundation of China (11025315, 10921303, 10973019, 11003024, 40890161, 11203037 and 41074123) and the CAS Project KJCX2EW-T07.

\section{References}

Kosovichev, A. G. 2011, ApJ, 734, L15

Schou, J., Scherrer, P. H., Bush, R. I., et al. 2012, Solar Phys., 275, 229

Song, Q., Zhang, J., Yang, S., \& Liu, Y. 2012, Research in Astronomy and Astrophysics, accepted, arXiv:1209.6192

Wang, J. 1999, Fundam. Cosmic Phys., 20, 251

Zhang, J., Li, L., \& Song, Q. 2007, ApJ, 662, L35 